\title{
RESPONSE OF HIGH RESOLUTION DRIFT TUBES TO RELATIVISTIC HEAVY IONS
}

\author{
A. TOMASCH, S.P. AHLEN * and C. BOWER \\ Phisics Department. Indiana Lineersity, Bloomington, Indiana 4740.5. USA
}

\section{P.B. PRICE and M.H. SALAMON}

Phisics Department, Liniversity of California. Berkeley, California 94720. USA

\section{G. TARLE and J. MUSSER}

Physics Department, Lnicersity of Michigan, Ann Arbor, Michigan 48109. USA

\author{
H. CRAWFORD \\ Space Sciences Lahoraton; Lniversity of California, Berkeley: California 94720, LSA
}

Received 15 May 1985

We report the results of various tests undertaken in a program to develop a drift tube hodoscope for a cosmic ray balloon experiment intended to search for extragalactic antimatter. Included are studies of mechanical integrity, electron drift velocity. tube gain. space charge saturation effects as measured for relativistic iron nuclei, and delta ray backgrounds associated with signals from iron projectiles. Implications of the results of these studies with regard to the use of drift tubes on balloon borne experiments are discussed. It is found that a spatial resolution of $\sigma \sim 300 \mu \mathrm{m}$ can be achieved over a dynamic range from $Z=20$ to 30 with little degradation from delta ray effects for suitably chosen tube gains and discriminator threshold settings.

\section{Introduction}

We have described in an earlier paper [1] an experiment (EXAM) designed to search for extragalactic antimatter in the cosmic rays. This balloon-borne experiment will use a novel combination of particle detectors (organic scintillators, Cherenkov counters, and CR-39 track etch detectors) to distinguish heavy cosmic ray nuclei from antinuclei. In addition, a hodoscope will be required to associate events measured in the etch detectors after a flight with the signals recorded in the active detectors during the flight. To reduce the number of ambiguous assignments to an acceptable level, and to adequately correct for particle zenith angle variations, requires a tracking resolution of $\sigma \sim 1 \mathrm{~mm}$, as was shown in ref. [1]. Our reasons for choosing drift tubes as the EXAM hodoscope were outlined in ref. [1]. Briefly, their small level of electromagnetic noise, their combination of mechanical durability and relative insensitivity to zenith angle effects, their extremely good intrinsic resolution and the availability of high density programmable time digitizing systems make them preferable to

* Alfred P. Sloan Research Fellow. such systems as spark chambers, multiwire proportional chambers and conventional drift chambers.

The matter-antimatter separation technique utilized by EXAM involves the charge sign asymmetry terms in the Mott cross section. This technique increases in efficacy with increasing atomic number, and has an effective threshold at silicon. Thus, the relatively abundant iron peak elements in the cosmic rays form a logical group to sample. However, the adaption of drift tube technology to the tracking of heavy ions presents problems not ordinarily encountered in high encrgy physics applications. In particular, delta ray background and space charge saturation drastically reduce the signal to noise ratio for heavy ion drift tube signals unless suitable precautions are taken. In the following sections we will describe these precautions. In addition. we will describe a number of other tests we have performed which characterize the performance of drift tubes in a balloon-borne experimental environment.

\section{Instrument description}

We have made a small prototype detector to aid in the design of our flight system. The prototype array 


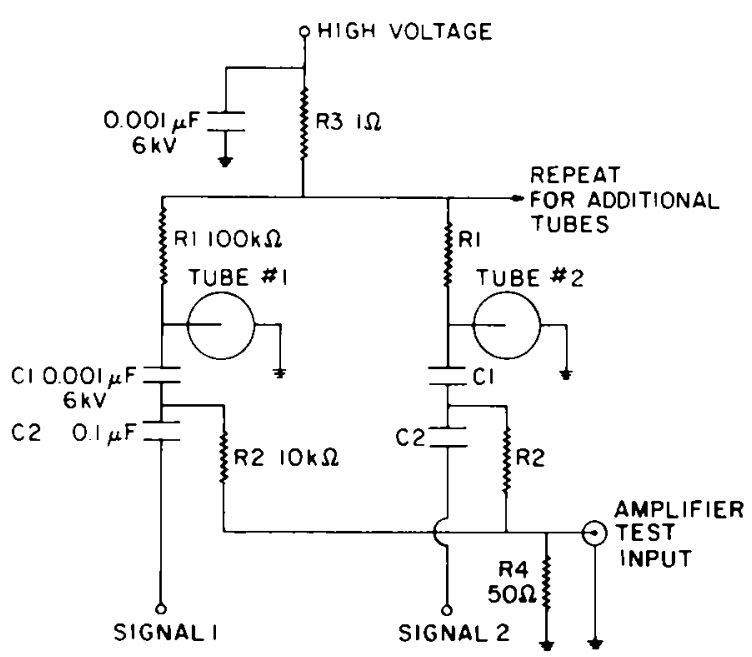

Fig. 1. Schematic for drift tube voltage divider network.

consists of eight stainless steel tubes, each being $30.5 \mathrm{~cm}$ long. These tubes have an inside diameter of $2.54 \mathrm{~cm}$ and a wall thickness of $152 \mu \mathrm{m}$. A $51 \mu \mathrm{m}$ diameter beryllium-copper $(\mathrm{Be}-\mathrm{Cu})$ alloy wire is strung down the center of each tube under a tension of $0.98 \mathrm{~N}$. Each end of each wire is precisely centered by passing it through a tiny steel ferrule (the "flea") which in turn fits into a slightly larger, and longer steel tube (the "flea collar"). The flea collar fits within a delrin pin which isolates the wire from ground and the delrin pin fits into a brass plug which is press fit into an aluminum block which is common to one end of all tubes. The drift tubes through which the wires pass are fit over brass plugs which are press fit into the aluminum end blocks. The two end blocks are then bolted to an aluminum plate base. Passages drilled in the end blocks provide gas consisting of $90 \%$ argon, $9 \%$ carbon dioxide and $1 \%$ methane to each tube. The methane is needed to quench photons which would otherwise cause the photoemission of electrons from the interior surfaces of the drift tubes. The divider network shown in fig. 1 applies positive high voltage to the sense wires. The design and construction of our tubes follow closely those of the outer drift tube modules used in the high resolution spectrometer (HRS) at PF:P [2]. A photograph of our prototype appears in fig. 2

\section{Time to space function}

The determination of the position of a particle from the drift time (which is what is measured in any experiment) requires knowledge of the so-called time to space function. This relates the time required for a liberated electron to drift from a given position to the wire. The time to space function for our tubes having an applied

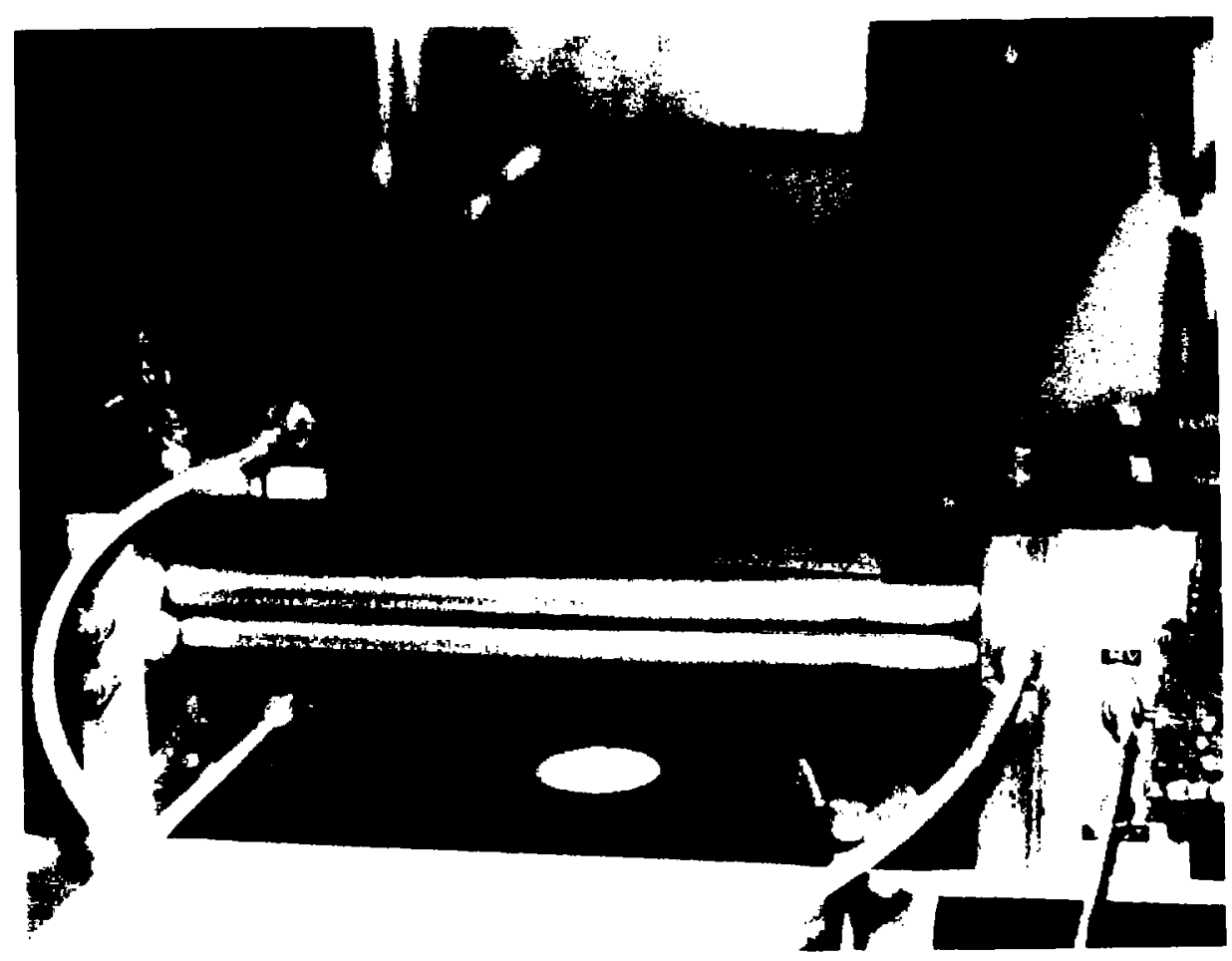

Fig. 2. Photograph of completed prototype drift tube array. 


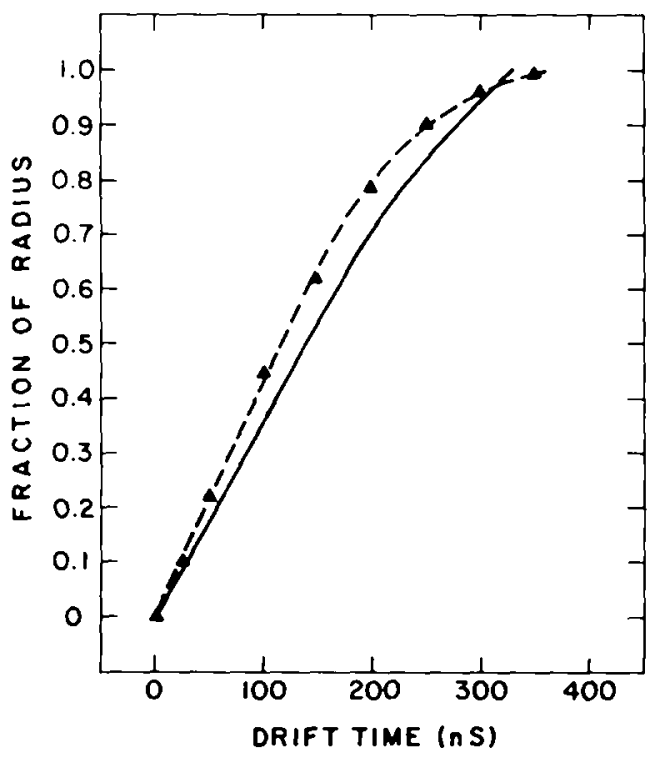

Fig. 3. Time to space curves for $2.54 \mathrm{~cm}$ drift tubes at $2200 \mathrm{~V}$ with a wire diameter of $51 \mu \mathrm{m}$. The gas mixture was $90 \%$ Ar, $9 \% \mathrm{CO}_{2}$ and $1 \%$ methane. The solid curve was computed using data from ref. [3]. The dashed curve is a measurement with our drift tube protolype.

voltage of $2200 \mathrm{~V}$ is shown in fig. 3. The dashed curve has been determined by us for our prototype in the following way. A pair of scintillators was set up to act in coincidence in such a way to select cosmic ray muons passing through some of our drift tubes (see fig. 4a). The coincidence generated a start signal for an Ortec 473 time to pulse height converter (TPHC). The stop signal was generated by one of the drift tubes. The drift tube output was first fed to a charge sensitive preamplifier (CSA) which has an integrating time of $10 \mu \mathrm{s}$ and a rise time of $10 \mathrm{~ns}$. The output of the CSA went to a fast (100 ns time constant) bipolar shaping amp (FSA) which went to the discriminator which generated the stop signal. The discriminator threshold was set about 50 times lower than the typical muon signal. The TPHC signal was binned by a LeCroy 3511 pulse height analyzer (PHA). The pulse height spectrum is the drift tube timing spectrum $N(t)$, where $N(t) \mathrm{d} t$ is the number of counts for drift times between $t$ and $t+\mathrm{d} t$. If one assumes the flux of muons to be parallel and uniform across the drift tube it follows that:

$\frac{r(t)}{b}=\frac{1}{N_{\mathrm{r}}} \sum_{t^{\prime}=0}^{\prime} N\left(t^{\prime}\right)$,

where $r(t)$ is the drift distance, $b$ is the inner radius of the tube, and $N_{\mathrm{T}}$ is the area under the $N(t)$ spectrum. The results appear as the dashed curve in fig. 3.

The solid curve in fig. 3 has been evaluated by using data [3] (see fig. 5) for the drift velocity of electrons as a

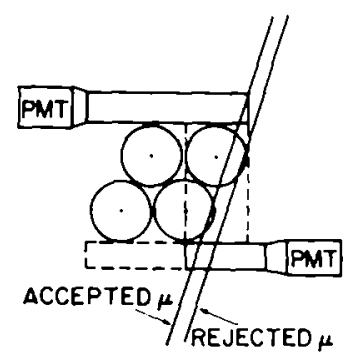

(o)

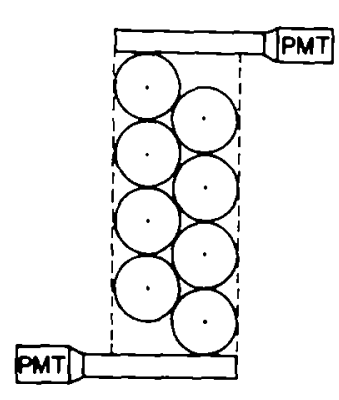

(c)

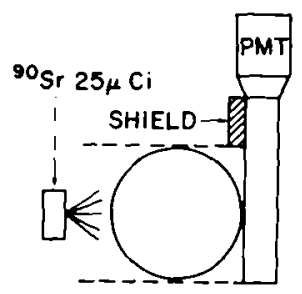

(b)

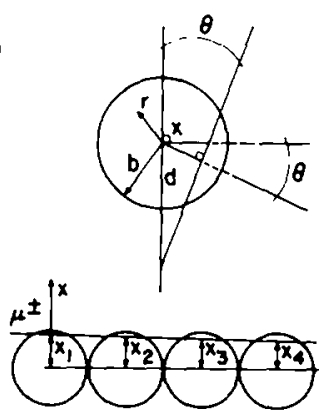

(d)
Fig. 4. Trigger geometries for (a) time to space measurements (the dashed lower scintillator outline shows the configuration for the muon gain measurements), (b) electron gain and temperature tests. and (c) the resolution study. Section (d) shows the geometry used for the resolution measurements. Note that $r$ denotes a radial position. while $d$ denotes the distance of closest approach to the wire for a particle traversing the tube.

function of electric field for a gas mixture identical to ours. The field for a cylindrical geometry is particularly simple:

$E(r)=\frac{V}{r \ln (b / a)}$.

where $V$ is the applied voltage and $a$ is the wire radius. With this equation and the data in fig. 5 the time to space function is evaluated by numerically integrating the function

$t(r)=\int_{a}^{r} \frac{d r^{\prime}}{w\left(r^{\prime}\right)}$,

where $w\left(r^{\prime}\right)$ is the drift velocity at radius $r^{\prime}$.

Of the two curves in fig. 3, the calculated time to space function is a more accurate reflection of the true tube response. The zero slope exhibited by the measured curve at $r=b$ implies zero drift velocity for electrons at the tube wall. This is clearly unphysical. The field strength at the wall is $279 \mathrm{~V} / \mathrm{cm}$. For this field strength, and electron accelerates to $-2 \mathrm{~cm} / \mu \mathrm{s}$ in about $0.1 \mathrm{~ns}$. The disagreement of the two curves suggests that the assumption of a plane parallel muon flux is a poor approximation. Muons incident at an angle to the vertical are accepted if they pass near the center of the tube 


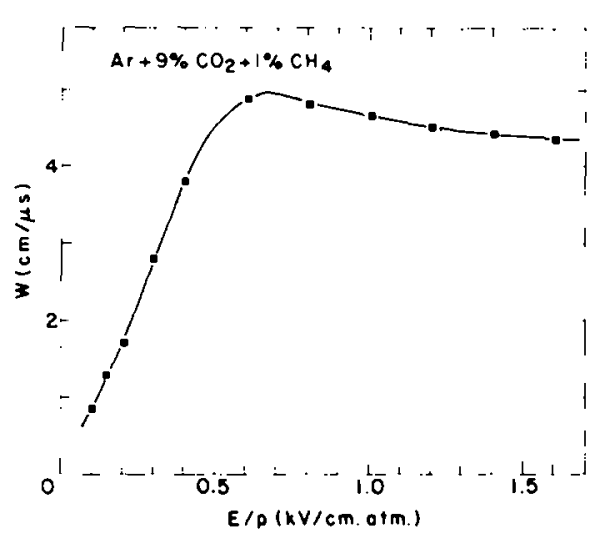

Fig. 5. Drift velocity as a function of reduced elcctric field for a gas mixture of $90 \% \mathrm{Ar}, 9 \% \mathrm{CO}_{2}$ and $1 \%$ methane from ref. [3].

but rejected if they pass near the tube edge for this trigger geometry, thus underpopulating the timing spectrum at late drift times (see fig. 4a). We plan more refined measurements to determine accurately the time to space function, as this will be quite important for locating the correct events in the CR-39 for EXAM.

\section{Tube gain}

We have measured drift tube gain both with muons and with electrons from a ${ }^{91} \mathrm{Sr}$ beta source $(25 \mu \mathrm{Ci})$. The muon data were taken with the scintillator trigger arrangement shown in fig. 4a providing the start signal for a gate generator. The drift tube signals from the four tubes shown were first integrated with CSAs and then summed with an Ortec 533 summing amplifier. The summed output was fed to an Ortec 573 shaping amplifier and the shaped pulse was directed to the input of the LeCroy 3511 PHA. The gate generator output pulse gated the PHA in coincidence mode. The charge delivered at the sense wire for a single muon within the (SA integration time $(\sim 1 / 2$ of the total charge collected) was determined from the peak channel of the muon pulse height distribution. Dividing the estimated total charge arriving at the wire by the initial charge deposited for a single muon yielded the gain value for a given sense wire voltage. These data are shown as the triangles in fig. 6.

The electron data (shown as squares in fig. 6) were obtained in a similar manner using a single tube and the trigger geometry shown in fig. $4 \mathrm{~b}$. Note that for this configuration, only electrons emitted by the strontium daughter ${ }^{90} \mathrm{Y}$ have sufficient range to penetrate the tube and trigger the scintillator (kinetic encrgy $>0.9 \mathrm{MeV}$ ). The high source activity caused a steady state distribution of space charge within the tube, weakening the electric field in the vicinity of the wire sufficiently to

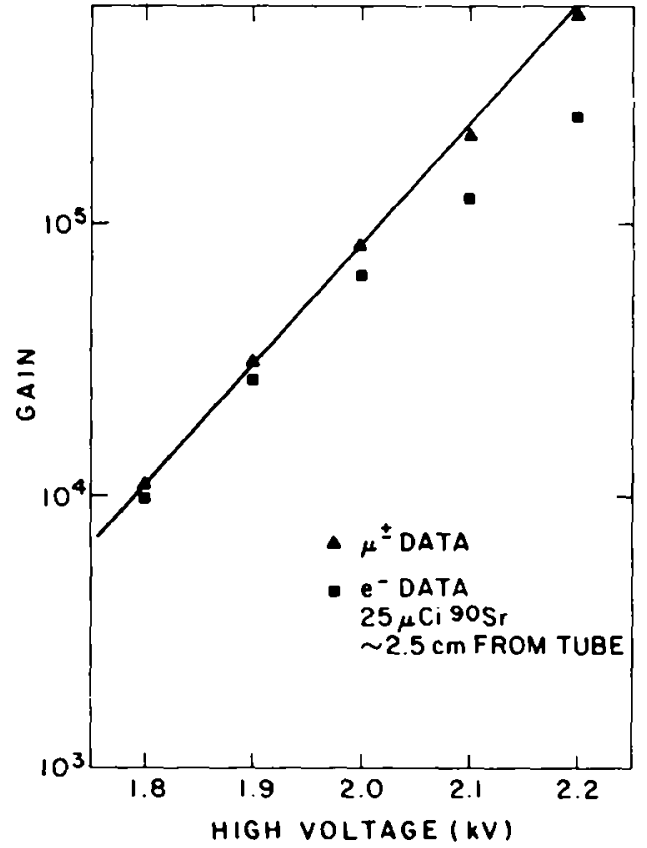

Fig. 6. Gain vs high voltage for a $51 \mu \mathrm{m}$ diameter wire in a 2.54 $\mathrm{cm}$ diameter drift tube with a mixture of $90 \% \mathrm{Ar}, 9 \% \mathrm{CO}_{2}$ and 1\% methane. The solid squares were obtained with electrons from a $25 \mu \mathrm{Ci}{ }^{90} \mathrm{Sr}$ source located $2.54 \mathrm{~cm}$ from the tube wall. The solid triangles were obtained with cosmic ray muons. Space charge effects account for the difference between the two sets of data. The solid line is the hest fit for the model described in the text.

reduce the amount of charge multiplication occurring as the electrons drifted to the wire. This is the cause of the discrepancy between the beta and muon data at higher voltages. Additional evidence for dose related saturation appears in fig. 7. For these measurements. current drawn by the tube from the high voltage supply was monitored as a function of wire voltage with the Sr source at two different distance $(1.3$ and $10 \mathrm{~cm})$ from the wire. The net current drawn (total current minus quiescent current with the source removed) at a given tube voltage reflects the tube gain since:

$$
\frac{\mathrm{d} q}{\mathrm{~d} t}-\frac{\mathrm{d} q_{0}}{\mathrm{~d} t}=e-\frac{\mathrm{d} N_{\mathrm{p}}}{\mathrm{d} t} M(V) .
$$

where $\mathrm{d} q / \mathrm{d} t\left(\mathrm{~d} q_{0} / \mathrm{d} t\right)$ is the tube current (quiescent current). $\mathrm{d} N_{\mathrm{p}} / \mathrm{d} t$ is the number of source related ionization electrons liberated within the tube per unit time. and $M(V)$ is the tube gain at wire voltage $V$. For the low dose data the net current increased by a factor fo 12 over the voltage range 1.8 to $2.2 \mathrm{kV}$. while the high dose current increased only a factor of 3.8 . In comparison. muon gain increased by a factor of 12.3 over the same range. The high dose data imply strong space charge effects, while the low dose points are relatively 


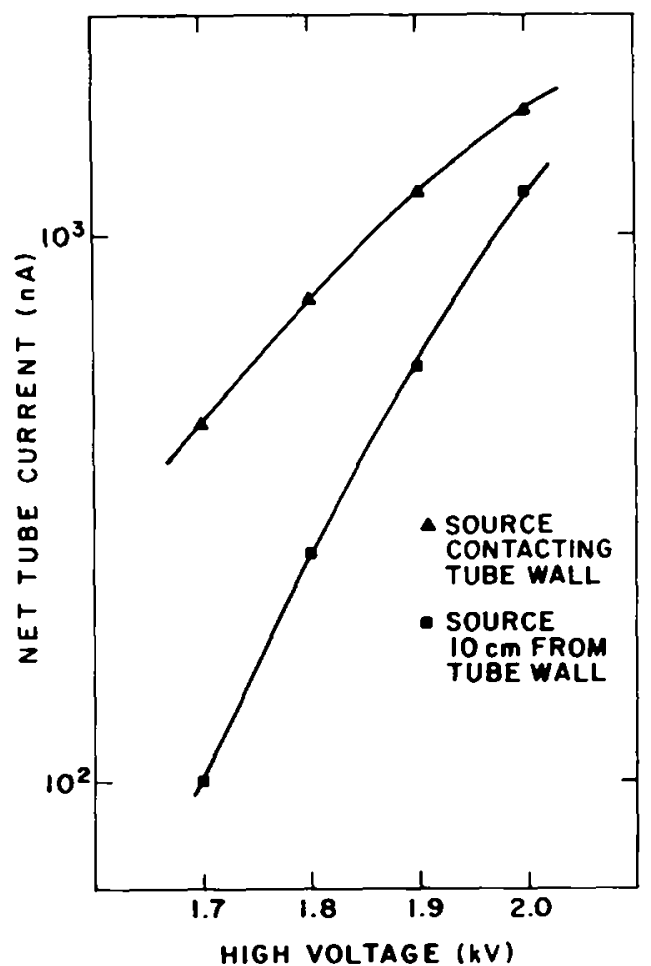

Fig. 7. Net tube current for electrons from a $25 \mu \mathrm{Ci}{ }^{90} \mathrm{Sr}$ source. The triangles are for the source in contact with the tube wall. The squares are for the source a distance $10 \mathrm{~cm}$ from the tube wall. The difference reflects space charge saturation effects

unsaturated. Both graphs display definite curvature, rather than the expected straight line characteristic of an exponential gain dependence [4], further suggesting that space charge effects are present for both dosages.

The solid line in fig. 6 represents our calculated best fit to the muon data using the simple model for gas multiplication presented by Sauli [4]. The gain for a proportional tube is:

$M(V)=\exp \left[\int_{a}^{r_{c}} \alpha(r) \mathrm{d} r\right]$,

where $\alpha$ denotes the first Townsend coefficient which is the inverse mean free path for electron impact ionization in the gas. The quantity $r_{\mathrm{c}}$ is defined as the radius at which the field strength becomes strong enough for gas multiplication to commence. Using a simple model for $\alpha$ :

$\alpha=k N_{\mathbf{\epsilon}}$,

where $N$ denotes the atomic number density of the gas, $\epsilon$ the potential difference through which an electron falls in one mean free path $(=E(r) / \alpha)$, and $k$ a constant, it follows from eqs. (2) and (6) that:

$\alpha(r)=\sqrt{\frac{V}{r} \frac{k N}{\ln (b / a)},}$ and hence from eq. (5) that:

$\ln M(V)=2 \sqrt{\frac{k N a}{\ln \left(\frac{N / a}{b}\right)}} \sqrt{V_{0}}\left[\sqrt{\frac{V_{0}}{V_{\mathrm{T}}}}-1\right]$.

where $r_{\mathrm{c}}$ has been eliminated in terms of $V_{\mathrm{T}}$. the threshold voltage at which amplification begins in the gas $\left(V / V_{\mathrm{T}}=r_{\mathrm{c}} / a\right)$. Note that for $V \gg V_{\mathrm{T}}$ :

$M(V)=K_{1} \exp \left(K_{2} V\right)$.

where $K_{1}$ and $K_{2}$ denote constants.

The best fit line shown in fig. 6 was obtained for $k=4 \times 10^{-18} \mathrm{~cm}^{2} / \mathrm{V}$ and $V_{\mathrm{r}}=751 \mathrm{~V}$. Note that this fit cannot be extrapolated over wide ranges of tube voltages since $\alpha$ varies strongly with $E / P$. Hence, a unique value for $k$ cannot be determined for a wide range of operating voltages.

\section{Spatial resolution}

In order to localize heavy ion tracks in the CR-39 track etch detectors flown aboard the FXAM experiment, the hodoscope must exhibit a spatial resolution (in terms of standard deviations) of $1 \mathrm{~mm}$ or less. To check our prototype, timing start and stop signals were generated in the same manner as for the time to space evaluation using the trigger geometry shown in fig. $4 \mathrm{c}$. A common start signal was generated by the scintillator coincidence and stop signals were generated by individual drift tube signals. The start and stops were directed to a LeCroy 2228A octal time to digital converter (TDC). In addition, the scintillator coincidence triggered a gate pulse generator which gated a LeCroy 2259A peak sensing analog to digital converter (ADC). The ADC recorded the fast shaping amplifier output pulse height to allow a timing walk correction to be applied to the raw TDC stop time. In this way, the timing errors introduced by the variations of the FSA pulse height relative to the discriminator threshold used to produce the TDC stop were removed. A LeCroy 3500 data acquisition system took the data automatically via CAMAC as directed by a FORTRAN acquisition program, storing the TDC and ADC channels for each of the eight tubes on a floppy disk.

To determine the one tube spatial resolution we followed the procedure below:

(1) Events were selected for which four vertically adjacent tubes were hit, with no hits in the other tubes.

(2) We required $|T D C-500| \leqslant 100$ channels where:

TDC $=\frac{1}{4} \sum_{i=1}^{4} \mathrm{TDC}_{i}$

which constrained $0.43 \leqslant(d / b) \leqslant 0.63$, where $d$ is the distance of closest approach for the muon to the wire (see fig. 4 d).

(3) For each hit tube, we required $\left|T D C_{1}-T D C\right| \leqslant$ 


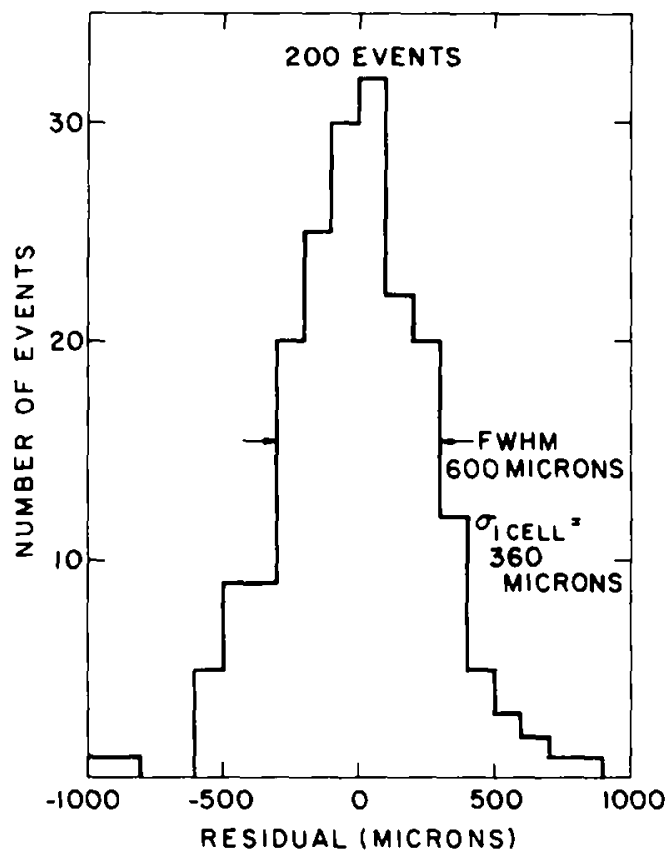

Fig. 8. Histogram of deviation of best fit straight line from data for muon trajectories through four drift tubes. See text for discussion.

100 channels which implied $|(d / b),-(d / b)| \leqslant 10^{1}$ and limited the maximum possible zenith angle for the incoming muon to $-10 \mathrm{mrad}$.

(4) Assuming $x_{t} \approx d_{\text {t }}$ for the coordinate system shown in fig. $4 \mathrm{~d}$. we computed a best fit straight line through the four measured points.

(5) We binned the residual deviation from the best fit line for each data point.

A histogram of the residuals appears in fig. 8. Taking into account the two degrees of freedom lost to the linear fit for each four tube event we obtain:

$\sigma_{1 \text { cell }}=360 \mu \mathrm{m}$.

This corresponds to a spatial resolution 2.8 times better than that required for the experiment. Unfortunately. this result does not reflect the true single cell resolution possible for tubes of this type, owing to poor machine work during production of the prototype endplates. Typical machining errors of $130-250 \mu \mathrm{m}$ exist in the sense wire positions, degrading the spatial resolution for the device considerably. Highly precise endplates for the balloon flight have been made and will be used to produce the next array to be used in further tests during the summer of 1985 . These parts, produced on a numerically controlled milling machine in the Physics Department machine shop at Berkeley, will maintain a wire placement spatial accuracy of $25 \mu \mathrm{m}$. The new tests will include actual tracking and location of etch pits for ion nuclei in C.R-39 with drift tubes, to verify that adequate resolution can be achieved with the $(R$ $39 /$ drift tube combination for heavy ions.

\section{Bevalac experiments}

It is well known that hodoscopes based on multiwire proportional chambers or on the closely related drift chambers are subject to problems for applications in which trajectories of relativistic heavy ions are to be determined $[5,6]$. This is due to two unrelated but mutually exacerbating phenomena: (1) relativistic heavy ions are accompanied in their passage through a detector by a large number of energetic, knock-on electrons (delta rays): what distinguishes heavy ions from singly charged projectiles is not the fraction of delta rays hut the mean number of them: for example as will be seen later, about 20 delta rays accompany a $1 \mathrm{GeV} / \mathrm{amu}$ iron nucleus as it passes through one of our drift tubes for each iron. whereas only one out of every 35 protons moving at the same velocity as the iron would be accompanied by one delta ray: and (2) proportional tubes are extremely vulnerable to space charge saturation effects which effectively reduce the tube gain for high ionization densities: we have seen in an earlier section how this manifests itself in the presence of a high generalized dose rate; but it also is important on an event by event basis for heavy nuclear projectiles: Goulding and Harvey have noted [7] that nonlinear effects are likely to set in when the product of the wire gain and energy loss of an ion in the chamber exceeds $200 \mathrm{MeV}$. Since a $1 \mathrm{GeV} / \mathrm{amu}$ iron nucleus deposits about $5 \mathrm{MeV}$ in a $2.54 \mathrm{~cm} \mathrm{Ar}$ drift tube, it is clear from fig. 6 that operating voltages considerably smaller than $1800 \mathrm{~V}$ must be used for linear operation. Ordinarily. one is not particularly concerned about linearity for timing operations. However, the delta rays are not subject to space charge limitations so that at large enough voltages the signal due to these can actually exceed that duc to the ion trail formed by the heavy nucleus. All timing information would be most likely lost in this case.

To investigate this problem we have taken our prototype chamber to the Lawrence Berkeley Laboratorys Bevalac to expose it to relativistic iron nuclei. Two experiments were performed. In each case two scintillator paddles were placed in front of the drift tubes and were set up in coincidence mode to select iron nuclei which passed through the tubes and to generate a start signal to evaluate timing characteristics. The outputs of the drift lubes each went to a LeCroy TRA401 fast linear amplifier, each of which was followed by a variable gain fast linear amplifier. The TRA401 has an input impedance of $(75 \pm 25) \Omega$ and a gain of $(12.5 \pm 2.5)$ $\mathrm{mV} / \mu \mathrm{A}$ when the output goes into $50 \Omega$. The variable gain amplifier has an input impedance of $50 \Omega$, an 
output impedance of about $10 \Omega$ and a gain variability from 1 to 10. All of the amplifiers used had pulse risetimes of no more than several ns. The outputs of the final amplifier stages were terminated by $50 \Omega$ and directed to the input of a Gould 4500 waveform digitizer. which was used to make permanent records of drift tube pulse shapes which were sampled every $10 \mathrm{~ns}$. The first of our two experiments used $800 \mathrm{MeV} / \mathrm{amu}$ iron nuclei and drift tube voltages from 1700 to $2400 \mathrm{~V}$. The second run used $1.2 \mathrm{GeV} / \mathrm{amu}$ iron and tube voltages from 1350 to $1700 \mathrm{~V}$.

The morphology of a typical iron pulse (for a wire voltage of $1500 \mathrm{~V}$ ) can be seen in fig. 9. The time scale is set so that $t=0$ corresponds to the time of passage of the iron nucleus through the tube. For this figure and all other data presented in this section, projectiles passed perpendicular to the wire in the drift tube, but at varying distances from the wire. Delta rays are produced at the tube walls and in upstream material when an iron nucleus passes through the chamber. The delta rays, in turn, cause ionization along their paths and some of the resultant drift electrons arrive at the wire before the electrons associated with the primary ionization trail of the iron. These early electrons give rise to what we call the delta ray plateau (see fig. 9). The height of this plateau appears to depend on the iron's distance of closest approach to the wire, with more delta rays entering the chamber when a nucleus just grazes the tube wall. This is consistent with the argument that the number of delta rays entering the chamber depends on the path length of the nucleus in the tube wall (see fig. 10). For grazing incidence, the nucleus has a much larger path length in the tube wall. Furthermore, the abundant low energy delta rays which are emitted at nearly right angles to the iron trajectory can enter the tube more easily for projectiles moving tangential to the thin steel walls than if they strike the tube wall head-on.

Following the delta ray plateau is the iron peak. This is caused by the arrival at the wire of drift electrons created in the region of the iron path which is closest to the wire. Electrons from the more distant parts of the path then arrive and form the iron plateau. The signal (which we will refer to as the reflection tail) following the iron plateau is not well understood. One possible explanation is that not all of the ultraviolet (UV) photons are quenched by the carbon dioxide and methane, and a small amount of photo-ionization takes place at the tube wall (so that this region of the pulse is a reflection of the iron peak and plateau).

To understand why the drift electrons which begin closest to the wire cause a larger signal than those formed near the tube wall, assume for the moment that the drift velocity is a constant throughout the tube. Fig. 11 shows isochrones (contours of equal drift times) separated by constant time intervals for this assumption. Note that a larger fraction of the nuclear path lies

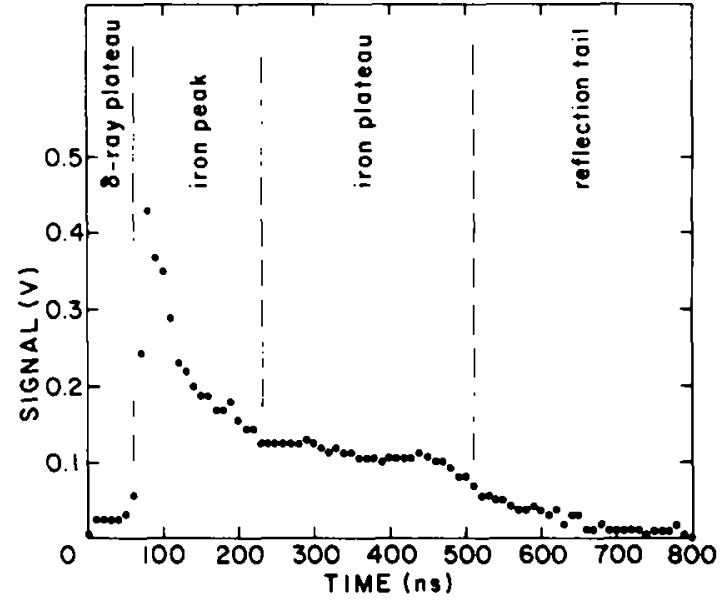

Fig. 9. A typical iron pulse for a wire voltage of $1500 \mathrm{~V}$. Note the four morphological regions.

in region 3 and thus, the portion of the pulse corresponding to this time interval is larger than for the other time intervals. The path lengths in the regions 4 through 6 are approximately the same, thus giving rise to the iron plateau. If a nucleus passes very near to or through the central wire, the path is equally divided among the isochronal regions and a flat pulse results.

For a given wire voltage, the variation of iron peak heights with the distance of closest approach between the trajectory and the wire is not severe. We show in fig. 12 five iron pulses having different iron peak drift times. These were taken at a wire voltage of $1500 \mathrm{~V}$ for $1.2 \mathrm{GeV} / \mathrm{amu}$ iron nuclei. The post-TRA401 amplifier gain was set at 4 . Note that these five events cover the entire temporal range from early (near wire passes: the leftmost peak) to very late (near wall passes: the rightmost peak). Although the late hits have more severe

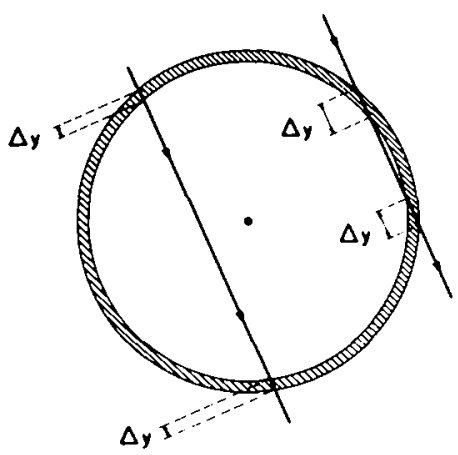

Fig. 10. A comparison of the length of tube wall traversed for near wire events and near wall events. The number of delta rays entering the chamber is larger for the near wall events due to the increased pathlength within the wall. and the more favorable geometry for the escape of low energy delta rays. 


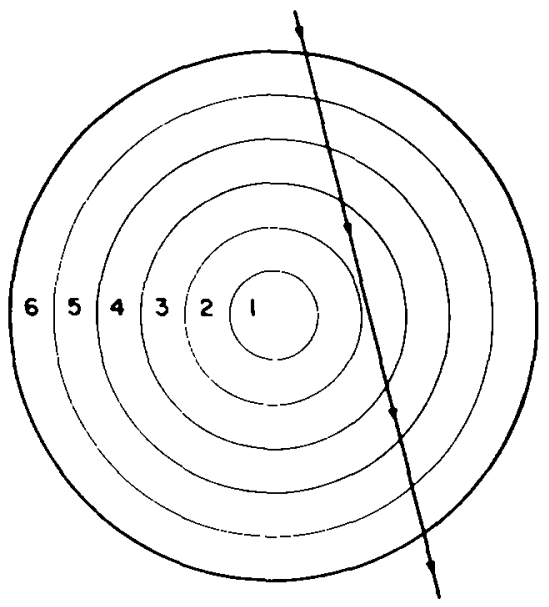

Fig. 11. An illustration of why there is a peak in the iron pulse. Rigions 1 through 6 are iscochrones. The path length in region 3 is greater than that in any other region.

delta ray problems it is clear that acceptable thresholds exist which could trigger on the iron peak without being interfered with by the delta ray plateau.

The number of delta rays contributing to the late hits has been evaluated by comparing the delta ray plateau at $1800 \mathrm{~V}$ for $800 \mathrm{MeV} / \mathrm{amu}$ iron with typical muon pulse peak heights at $2200 \mathrm{~V}$. taking the measured gain variation into account. By scaling delta ray production by $1 / \beta^{2}$ ( $\beta$ being the projectile velocity in units of the speed of light) it has been inferred that the delta ray plateau for the late hit in fig. 12 is 50 times the peak signal of a cosmic ray muon. Thus the delta ray plateau for the early hit in fig. 12 is about 10 times the level of a single muon. Since delta ray plateaus for early hits start about $60 \mathrm{~ns}$ prior to the iron peak and since the iron peak itself is about $60 \mathrm{~ns}$ wide, it is reasonable to conclude that about 20 delta rays accompany the iron

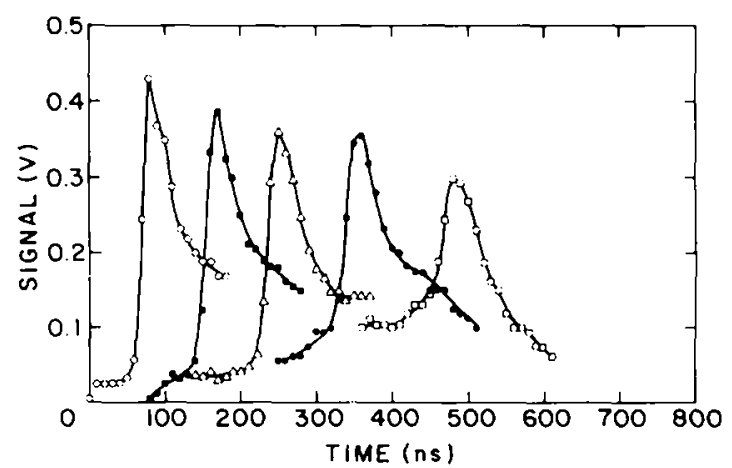

Fig. 12. A comparison of $1.2 \mathrm{GeV} / \mathrm{amu}$ iron pulse heights having different arrival times. The drift tube potential for these pulses was $1500 \mathrm{~V}$. nucleus for head-on collisions with the tube wall. This agrees reasonably well with a calculation we have done which estimates the number of delta rays which escape from a flat slab of matter upon which a heavy nucleus is normally incident. By following the same basic procedure carried out in ref. [8] to estimate energy carried away by escaping delta rays. we find that the number of excaping delta rays is:

$N=\frac{0.075 Z^{2}}{\beta^{4} \gamma^{2}}\left[2 \sqrt{ } T R+! \beta^{2} T\left(\ln \frac{T}{R}-1\right)-T\right]$.

where $Z(\gamma)$ is the atomic number (Lorenty factor) of the projectile, $T$ is the slab thickness and $R$ is the average range of the most energetic delta ray (both $T$ and $R$ are in $\mathrm{g} / \mathrm{cm}^{2}$ ). If $T>R$. one should replace $T$ with $R$ in eq. (12). For the Bevalac experiment at 1.2 GeV/amu. $T$ was approximately $2 \mathrm{~g} / \mathrm{cm}^{2}$ (this includes scintillators, wrapping material, etc. as well as the steel wall) and $R$ was $1.9 \mathrm{~g} / \mathrm{cm}^{2}$. This yields a value of $N=17$ delta rays, in excellent agreement with our observation. However, it should be noted that for the earliest hit in fig. 12 the iron peak is about 200 times larger than a single muon pulse. It should be about $26^{2}=676$ times larger in the absence of space charge saturation. This means that signal to noise can be improved by more than a factor of 3 over that shown in fig. 12 by reducing the drift tube gain further. In this respect it is worth noting that the product of iron energy loss and wire gain at $1500 \mathrm{~V}$ is about $3000 \mathrm{MeV}$. about 15 times larger than the recommended region for linear response.

A more dramatic demonstration of space charge effects occurred in our first Bevalac exposure at $2200 \mathrm{~V}$ for which there was not even a hint of an iron peak. It was completely obscured by the delta ray plateau. A somewhat more quantitative assessment of saturation effects can be obtained from fig. 1.3 where iron hits at

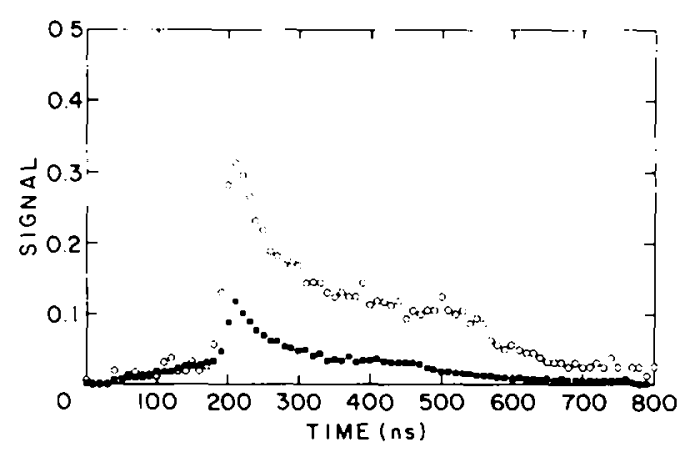

Fig. 13. A comparison of $1.2 \mathrm{GeV} / \mathrm{amu}$ iron pulses having different thamber potentials: $1350 \mathrm{~V}$ (circles) and $1700 \mathrm{~V}$ (squares). The curve are normalized to have the same delta ray plateaus. For $2200 \mathrm{~V}$ (not shown), the iron peak was not visible above the delta ray hackground. 
roughly the same distance from the wire are compared for wire voltages of 1350 and $1700 \mathrm{~V}$. The pulses have been normalized by setting their delta ray plateaus at the same arbitrary level. Signal to noise has been improved by nearly a factor of 3 . We expect that with high quality, high gain amplifiers (such as the LeCroy TRA403), our drift tubes can be operated at voltages as small as $1200 \mathrm{~V}$ for which delta ray levels should pose no significant problem for a dynamic range from calcium to nickel.

\section{Model for drift tube signal formation}

In this section. we present the details of a model we have formulated to account quantitatively for the features of the iron pulses discussed in the previous section. The geometry we rely on is shown in fig. 14. As a heavy ion passes through the drift tube, it leaves in its path a trail of electron-ion pairs. For a $1 \mathrm{GeV} / \mathrm{amu}$ iron nucleus there are on the order of $10^{5}$ of these pairs formed in a single tube. We assume that these electrons are uniformly distributed along this path and that all of them are formed at the same time (the flight time of a relativistic nucleus through the tube is on the order of $0.1 \mathrm{~ns}$; this is negligible compared to the intrinsic wire response time [4] of order $10 \mathrm{~ns}$ ).

Consider a packet of $\mathrm{d} N$ electrons which originate at position $x$ within a path-length interval $\mathrm{d} x$ (see fig. 14). They will travel towards the central wire along the path $z$ with a drift velocity which is a function of distance (see fig. 15 for the radial dependence of drift velocity as inferred from data in ref. [3]). We define the mean drift time. $t_{0}$ by the equation:

$t_{0}(x, d)=\int_{0}^{\sqrt{1}=d^{2}} \frac{\mathrm{d} r^{\prime}}{\mathrm{u} \cdot\left(r^{\prime}\right)}$.

where $d$ is defined in fig. 14 and $w^{\prime}\left(r^{\prime}\right)$ is the drift velocity at a distance $r^{\prime}$ from the wire.

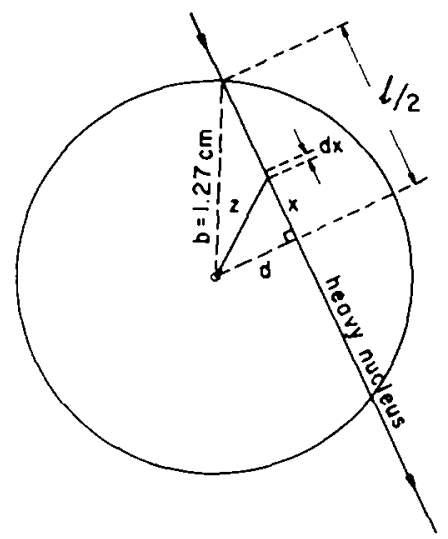

Fig. 14. The variables and dimensions used in the model for drift tube response.

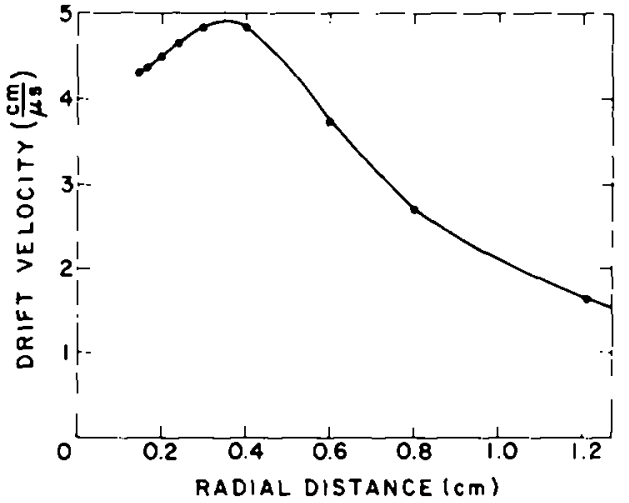

Fig. 15. Electron drift velocity as a function of distance from the central wire for a drift tube with a radius of $1.27 \mathrm{~cm}$, a potential of $1500 \mathrm{~V}$, and a gas mixture of $90 \% \mathrm{Ar}, 9 \% \mathrm{CO}_{2}$, and 1 to methane.

We now let $t=0$ designate the time of the heavy ion's passage through the tube. Assuming the distribution of arrival times within the packet is Gaussian with a mean value $t_{0}$ and a standard deviation $\sigma=10 \mathrm{~ns}$ (the intrinsic wire response time), the pulse current as a function of time and distance of closest approach to the wire $(d)$ is:

signal $(t, d) \propto \frac{\mathrm{d} N}{\mathrm{~d} x} \int_{1 / 2}^{l / 2} \mathrm{~d} x \exp \left\{\frac{\left[t-t_{0}(x \cdot d)\right]^{2}}{2 \sigma^{2}}\right\}$.

where $\mathrm{d} N / \mathrm{d} x$ is the linear density of primary electron-ion pairs along the particle's trajectory. It should be noted that if the path of the particle is not in a plane perpendicular to the wire, then the above equation still applies if we use a larger value of $\mathrm{d} N / \mathrm{d} x$. This generalization may be visualized by projecting the trajectory onto such a plane.

The above model was fit to the $1500 \mathrm{~V}$ data. Three free parameters were used: (1) a constant of proportionality for eq. (14) of 1.6: (2) a constant delta ray background level of 0.05 ; and (3) an attenuation factor. This factor was necessary to account for the observed variability of pulse heights and is probably due to the recombination of clectron-ion pairs in the outer (weak field) region of the tube. It was found to be given by:

$A(z)=\exp (-0.28 z), \quad 0 \leq z \leq 1.27 \mathrm{~cm}$.

At the tube edge. about $30 \%$ of the electron-ion pairs are lost to whatever mechanism is responsible for the attenuation.

We compare this model with experimental pulses in fig. 16. A discrepancy between 400 and $500 \mathrm{~ns}$ can be seen. This may be due to the fact that the drift velocity data [3] used in the model were obtained for singly charged particles. There is an apparent difference between the maximum drift time derived from the data in 


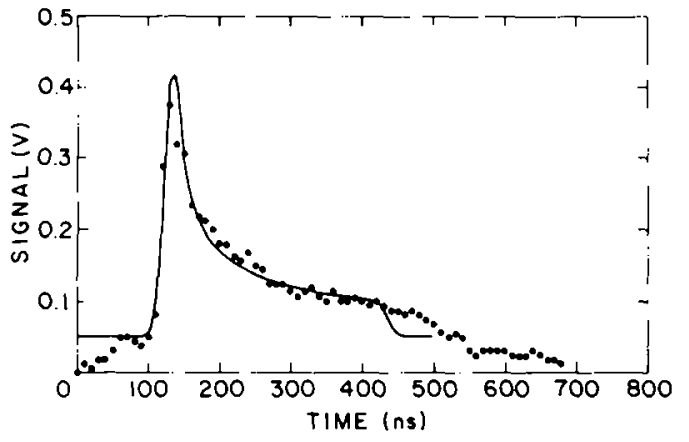

Fig. 16. A comparison of a $1.2 \mathrm{GeV} / \mathrm{amu}$ iron pulse (circles) with the theory. See the text for a more detailed discussion.

ref. [3] and that obtained for the iron pulses. The former is found to be $(435 \pm 20) \mathrm{ns}$, while the latter is $(510 \pm 10)$ ns (each being evaluated for a wire voltage of $1500 \mathrm{~V}$; the iron maximum drift time is defined to be the half maximum point of the falloff from the iron plateau to the reflection tail). The discrepancy may result from space charge affects, either due to a delta ray dose in the vicinity of the wire, or to the positive ion trail along the iron trajectory from which the electrons must migrate.

\section{Temperature stability}

The electron gain setup described in an earlier section was temperature eycled. using a specially constructed gas tight drift tube to check for temperature dependent gain changes at constant gas density. Pollution of the gas mixture by organic molecules from the epoxy used to assemble the detector might cause the tube gain to change at high temperatures, while condensates in the gas could affect the tube at low temperatures. The temperature in a balloon gondola can range from below freezing to over $40^{\circ} \mathrm{C}$, and cannot be easily predicted with great accuracy. Should severe temperature effects occur. discriminator thresholds set on the ground might be inappropriate for the flight. However. our tests have shown this not to be a problem. They indicate that tube gain is stable for times up to several hours for temperatures from 4 to $44^{\circ} \mathrm{C}$.

\section{Wire strength}

To verify that the sense wires will not suffer excessive breakage while the experiment is transported. launched, and recovered, we have performed extensive wire loading tests, determining the equivalent $g$ loading (applied acceleration in units of one earth gravitational acceleration) at which wires fail under a variety of wire schemes. We simulated an acceleration of $f\left(980 \mathrm{~cm} / \mathrm{s}^{2}\right)$ by increasing the wire mass per unit length by the factor $f$. This was accomplished by hanging a large number of small weights uniformly along the wire. We checked the validity of this technique as an approximation to a continuous increased mass per unit length by comparing the measured wire figure to a catenary with mass per unit length equal to the average value for the weighted wire. These measurements showed the weighted wire to be a reasonable approximation.

The loading tests indicated that a $51 \mu \mathrm{m}$ diameter $\mathrm{Be} \mathrm{Cu}$ alloy wire fails at typical accelerations of $1400 \mathrm{~g}$. while $51 \mu \mathrm{m}$ gold-plated tungsten wire fails at $2800 \mathrm{~g}$. Typical accelerations encountered during transportation and parachute deployment fall in the range of $(8-10) \mathrm{g}$. Since the wires exhibit such great intrinsic strength. maintaining them in a nick and kink free condition dominates all other considerations for preventing excessive wire failure.

\section{Acknowledgments}

We would like to express our appreciation to $T$. Roberts for assisting with the mechanical and thermal tests of the drift tubes, to H. Ogren and D. Rust for useful advice on drift tube construction. and to the staff of the LBL. Bevalac for their cooperation in our heavy ion exposures. This work was supported by a research fellowship from the Alfred P. Sloan Foundation and a grant from the National Science Foundation (NSF PHY 84-14186).

\section{References}

[1] S.P. Ahlen. P.B. Price, M.H. Salamon and G. Tarle, Niucl. Instr. and Meth. 485 (1982) 197.

[2] G. Baranko, PhI) Thesis, Indiana University (1983).

[3] I. Lehraus. R. Matthewson and W. Tejessy. Nucl. Instr. and Meth. 199 (1982) 200.

[4] F. Sauli. CERN Publ. p. 40 (1977) 7709.

[5] M. Simon. M. Henkel and (j. Schwieweck, Nucl. Instr. and Meth. 483 (1982) 192.

[6] M. Simon, M. Henkel, R. Hundt, K.1). Mathis, (i. Schwieweck and T. Suck. Nucl lnstr. and Meth. 466 (1984) 221.

[7] F.S. Goulding and B.G. Harvey, Ann. Rev. Nucl. Sci. 25 (1975) 167

[8] S.P. Ahlen. Rev. Mod. Phys. 52 (1980) 121. 